\title{
WINDUP PROPERTIES OF RECURSIVE PARAMETER ESTIMATION ALGORITHMS IN ACOUSTIC ECHO CANCELLATION
}

\author{
Magnus Evestedt, Alexander Medvedev and Torbjörn Wigren
}

\author{
Department of Information Technology, Uppsala University, \\ P. O. Box 337, SE-951 05, SWEDEN
}

\begin{abstract}
The windup properties of a recently suggested recursive parameter estimation algorithm are investigated in comparison with a number of well-known techniques such as the Normalized Least Squares Algorithm (NLMS) and the Kalman filter (KF). An acoustic echo cancellation application is used as a benchmark for comparing the properties of different approaches. The basic performance of the method, both for white and colored input signal, appears to be similar to that of the KF and superior to the NLMS. When the energy in the input signal decreases, the algorithm performs best of all compared estimation schemes. Once the solution of the Riccati equation of the algorithm converged to a user defined point, it will stay there even if the input excitation is reduced. This explains the good anti-windup properties of the method. Copyright ${ }^{\complement} 2005$ IFAC
\end{abstract}

Keywords: Acoustic echo cancellation, recursive estimation, windup

\section{INTRODUCTION}

Recursive parameter estimation is an integral part of many signal processing and control applications such as echo cancellation, active vibration control, fault detection and indirect adaptive control. Different methods have been considered in the past, for instance the Normalized Least Mean Squares (NLMS), Recursive Least Squares (RLS) and fast Kalman filter methods, see (Ljung and Gunnarsson, 1990).

Without stating a mathematical model for parameter variation, no recursive parameter estimation method can be proven to be better performance-wise than any other, (Ljung and Gunnarsson, 1990). The matter becomes even more complicated when robustness issues are taken into consideration. From an engineering point of view, it is more relevant to consider what algorithm suites best a particular application. Then, the nature of the application can provide necessary $a$ priori modelling information and also a useful benchmark for a fair and instructive comparison between different estimation approaches.

In this paper recursive parameter estimation for acoustic echo cancellation (AEC) is studied. The problem of AEC arises whenever a loudspeaker and a microphone are located so that the microphone picks up the signal from the loudspeaker, (Breining et al., 1999). The goal of AEC is to remove the overhearing from the loudspeaker into the microphone signal and thereby avoid- ing an echo at the transmitting end. An illustration of a typical AEC setup is given in Fig. 1. In the figure, $x(t)$ is the signal from the transmitting end and $e(t)$ is the returning signal to the transmitting end. The impulse response $h(n)$ describes the echo path including the loudspeaker acoustics and the microphone, while $\hat{h}(n)$ is the estimated impulse response. The local speech signal $s(t)$ and the local noise $v(t)$ constitute the additional inputs to the microphone. If the estimate $\hat{h}(n)$ is accurate, the echo $d(t)$ can be subtracted from the outgoing signal $y(t)$.

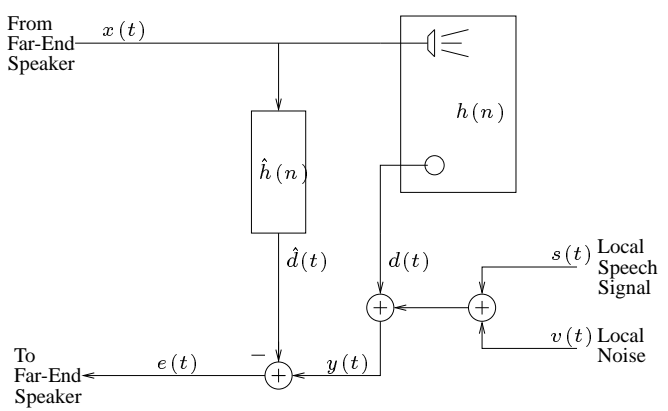

Fig. 1. Basic features of an acoustic echo cancellation system.

A finite impulse response (FIR) filter is normally used for modelling of the acoustic echo path and for 
prediction of the echoes. Experiments show that no detailed pole-zero structure can be a priori imposed on the impulse response of the channel. The FIR structure is thus chosen for its flexibility and to avoid stability problems. Usually, this choice results in a high order filter.

Since the algorithms are to be applied in real time, the computational complexity and the memory requirements of the algorithm should be kept reasonably low. It is also important that the algorithm adapts rapidly, when the echo paths change.

A speech signal is colored and sometimes fails to provide sufficient excitation for estimation of the filter parameters. When the excitation in the input signal is insufficient (the channel is silent), a phenomenon referred to as (covariance) windup occurs in the Kalman filter-based parameter estimation algorithm. Then some eigenvalues of the Riccati equation grow linearly in time until excitation is recovered.

Many methods aiming at prevention of the windup problem have been proposed in the literature, see (Gunnarsson, 1996; Cao and Schwartz, 2004) and references therein. Usually, speech detection algorithms are employed to detect whether the energy in the signal is large enough. If it is not, the estimation is turned off. A common problem with such algorithms is the choice of threshold for the filter adaptation. In principle, the threshold has to be adaptive to have effect in different acoustic environments. Another and more systematic way to overcome this problem is to have a parameter estimator that is insensitive to reduction of the input signal energy.

Recently, a version of the Kalman filter with improved windup properties was presented in (Stenlund and Gustafsson, 2002). This algorithm, in the sequel referred to as the Stenlund-Gustafsson (SG) algorithm, has the robustness of the NLMS and a convergence rate similar to that of the Kalman filter. The SGalgorithm is shown to be non-diverging under lack of excitation in (Medvedev, 2003; Medvedev, 2004). Steady state solutions are studied in (Evestedt and Medvedev, 2005).

The main focus of this paper is on studying the antiwindup properties of the SG algorithm in comparison to the NLMS algorithm, the Kalman filter and the algorithm suggested in (Cao and Schwartz, 2004).

As a benchmark for performance comparison of different parameter estimation methods, an AEC application is used. Simulations are performed with white noise and colored input (music), to evaluate basic performance measures of the algorithm. Then an example is given with a piecewise stationary input to highlight the importance of the choice of the tuning parameters in the SG algorithm. Next the windup properties are investigated by letting the energy in the input signal decrease over time and studying how the signal decay is reflected in the behavior of the algorithms. Finally simulations are run to test the performance of the SG algorithm in terms of tracking of the set point.

\section{STUDIED RECURSIVE PARAMETER ESTIMATION ALGORITHMS}

To describe the ideas briefly, consider the linear FIR model, in the regressor form,

$$
y(t)=\varphi^{T}(t) \theta+e(t)
$$

where $y(t)$ is the scalar output measured at discrete time instances $t=[0, \infty), \varphi \in R^{n}$ is the regressor vector containing current and past input values, $\theta \in$ $R^{n}$ is the parameter vector to be estimated and the scalar $e$ is the disturbance.

According to (Ljung and Gunnarsson, 1990), the typical structure of the recursive algorithm to estimate the filter parameters is given by,

$$
\hat{\theta}(t)=\hat{\theta}(t-1)+K(t)\left(y(t)-\varphi^{T}(t) \hat{\theta}(t-1)\right)
$$

where $K(t)$ is the adaptation gain.

A Kalman filter based approach to calculating the adaptation gain is,

$$
\begin{aligned}
& K(t)=P(t) \varphi(t) \\
& P(t)=P(t-1)-\frac{P(t-1) \varphi(t) \varphi^{T}(t) P(t-1)}{r(t)+\varphi^{T}(t) P(t-1) \varphi(t)}+Q(t)
\end{aligned}
$$

where $P(\cdot) \in R^{n \times n}, Q(\cdot) \in R^{n \times n}, Q(\cdot) \geq$ $0, P(0)=P^{T}(0), P(0) \geq 0, r(t)$ is a positive scalar and $t \in\{1,2, \ldots, \infty\}$.

Optimality of the estimate, $\hat{\theta}$, is guaranteed only when $\theta$ is subject to a random walk model where $Q$ is the covariance of the white process driving the random walk model and $r(t)=$ var $e(t)$, (Ljung and Gunnarsson, 1990). Since these quantities are seldom known $a$ priori, even when the random walk model is justified, they are usually treated as design parameters of the estimation algorithm and chosen to achieve some desired properties of the filter. For instance, the degrees of freedom in $Q$ and $r$ can be traded in (3), for better windup performance.

In (Stenlund and Gustafsson, 2002) a special choice of $Q(t)$ is used to control the convergence point of Riccati equation (3),

$$
Q(t)=\frac{P_{d} \varphi(t) \varphi^{T}(t) P_{d}}{r(t)+\varphi^{T}(t) P_{d} \varphi(t)}
$$

where $P_{d} \in R^{n \times n}, P_{d}>0$. Thus, the matrix $P_{d}$ becomes a stationary point of (3). Similarly, a directional tracking algorithm in (Cao and Schwartz, 2004), the one identified as Algorithm 1, makes use of the free term in (3) in the form,

$$
Q(t)=\frac{\gamma \varphi(t) \varphi^{T}(t)}{\epsilon+\varphi^{T}(t) \varphi(t)}
$$

where $\gamma>0$ and $\epsilon>0$ are some scalars. This algorithm is obtained as a special case of the SG algorithm by letting $\epsilon \gamma=r$ and $P_{d}=\gamma I$.

To economize on the demanding matrix computations in the Riccati equation, an Averaged Kalman Filter (AKFA) is developed in (Wigren, 1998). Estimating the parameters in (1), AKFA replaces certain variables with averages. This produces a small number of scalar Riccati equations with adaptation gains that can be pre-computed or computed online. The algorithm can be summarized as follows. 


$$
\begin{aligned}
& \hat{\sigma}^{2}(0)=0 ; \quad \bar{p}_{i}(0)=p_{i}(0) i=1, \ldots, n \\
& \hat{\sigma}^{2}(t)=\left\{\begin{array}{c}
{\left[\left(1-\frac{1}{t}\right) \hat{\sigma}_{N}^{2}(t-1)+\frac{N}{t} x^{2}(t)\right]_{1 \leq t \leq N}} \\
{\left[\hat{\sigma}_{N}^{2}(t-1)+x^{2}(t)-x^{2}(t-N)\right]_{t>N}}
\end{array}\right. \\
& \bar{p}_{i}(t+1)=\bar{p}_{i}(t)+S(t-i) q_{i}-\frac{S(t-i) \bar{p}_{i}^{2}(t)}{\alpha+S(t-i) \bar{p}_{i}(t)} \\
& K(t)=\frac{N}{\epsilon+\alpha \hat{\sigma}_{N}^{2}(t)}\left(\bar{p}_{1}(t) x(t-1) \ldots \bar{p}_{n}(t) x(t-n)\right)^{T} \\
& \varphi(t)=(x(t-1) \ldots x(t-n))^{T} ; x(t)=0 ; t>0
\end{aligned}
$$

where the $\bar{p}_{i}(t)$ are averaged diagonal elements from the Riccati equation and $q_{i}$ are the diagonal elements of $Q$. The parameter $N$ is the sliding window length and $\hat{\sigma}_{N}^{2}(t)$ is the estimate of the total input signal energy in the sliding window. The function $S(t)$ is the unit step function in $t=0$.

Since the experiments on AEC in this paper follow (Wigren, 1998), some properties related to the tuning of the AKFA are reproduced here. The initial choice of the $p_{i}(0)$ can be for example a piecewise constant exponential decay,

$$
p_{i}(0)=\beta e^{[i-1 / l]}, \frac{n}{l}=m \in Z_{+}
$$

Then the envelope of the impulse response is generally governed by a few dominant poles, which results in an exponential decay of the expected impulse response power. The number of piecewise constant intervals equals $m$ and [.] denotes the integer function.

If an upper bound on echo impulse response power (Power) is available, it follows that Power = $\sum_{i=1}^{n} p_{i}(0)$. Summing up using (5) gives,

$$
\beta=\left\{\begin{array}{l}
\frac{1}{n} \text { Power } ; m=1 \\
\frac{1}{l} \text { Power } \frac{1-e^{-\gamma}}{1-e^{1-e^{-m \gamma}}} ; m>1
\end{array}\right.
$$

where $\gamma$ can be determined by specification of the residual power at tap $n$ as compared to tap 1, using (5). If the residual power is specified by $\delta$ as $\beta e^{-\gamma(n-1) / l}=p_{n}(0)=\delta p_{1}(0)=\delta \beta$, it follows that,

$$
\delta=1, m=1 ; \gamma=-\frac{\log (\delta)}{m-1} m, m>1
$$

By choosing $\delta, n, m$ and Power, $p_{i}(0)$ can now be computed. The parameter $\alpha=f_{1} n \max _{i} p_{i}(0)$ where $f_{1} \geq 1$ follows

The $q_{i}$ are related to the average variation rate of the $i$ th filter tap via the random walk model. They can be determined as

$$
q_{i}=q_{1} e^{-\gamma[i-1 / l]}, i=2, \ldots, n
$$

The parameter $q_{1}$ is given by

$$
q_{1}=f_{2}^{2} \frac{\left(\min \left(\frac{2 \alpha}{n}, p_{1}(0)\right)\right)^{2}}{\alpha+f_{2} \min \left(\frac{2 \alpha}{n}, p_{1}(0)\right)}
$$

where $0<f_{2}<1$. Further information on tuning of the parameters can be found in (Wigren, 1998).

To get rid of the computational costs due to the Riccati equation, the NLMS recursion is often employed, $K(t)=\frac{\mu}{1+\varphi^{T}(t) \varphi(t)} \varphi(t)$, where $\mu \in R^{+}$is the adaptation gain. In (Ljung and Gunnarsson, 1990), it is shown that the NLMS can be obtained as a special case of the Kalman filter with the parameters,

$$
P_{d}=\alpha I, \alpha \in R^{+} ; P(0)=P_{d} ; r(t)=1
$$

in the equations (3), (4). In the NLMS, the Riccati equation becomes redundant since it is initiated at its stationary point. Thus, the resulting filter (2) becomes insensitive to loss of excitation. In fact, Algorithm 1 in (Cao and Schwartz, 2004) coincides exactly with the NLMS provided the Riccati equation is initiated as $P(0)=\gamma I$. Notice here that iterating the Riccati equation at each step significantly improves convergence rate of the NLMS in the case of non-stationary data.

Recapitalizing for the recursive parameter estimation algorithms, one can note that optimality of parameter estimates (minimum variance etc.) is seldom an issue. Performance wise the tradeoff is mostly between tracking abilities of the estimate and its robustness to lack of excitation. The inflicted computational cost and memory demand are as well highly important in real time applications.

When high convergence speed is desired, the data are persistently exciting and processor power is available, the Kalman filter is the best choice. Fast Kalman-type algorithms have been suggested, but many schemes show numerical stability problems, (Hänsler, 1992). If highest robustness and cheap implementation are the main priorities, the NLMS is probably the algorithm to go for. However, the choices in between are more difficult to make.

\section{PROPERTIES OF THE ALGORITHMS}

In (Wigren, 1998) the properties of the AKFA were compared to those of the Kalman filter and the NLMS. By using numerical examples, the AKFA was shown to perform significantly better than the NLMS, in terms of settling time to the same echo suppression level, for white inputs. In fact, the proposed scheme performed very close to the Kalman filter. For colored signals (music), the Kalman filter performed best. The AKFA, however, performed significantly better than the NLMS algorithm at expense of an negligible increase of the computational burden. However, the algorithms were not tested for a case of insufficient excitation.

The SG algorithm was developed especially for handling the windup phenomenon. It is possible to see, (Evestedt and Medvedev, 2005), that (3) can, both for symmetric and non-symmetric solutions, be written as,

$$
\begin{aligned}
P(t) & =A_{t}^{-1}(P(t-1)) P(t-1) A_{t}^{-T}\left(P_{d}\right) \\
& -A_{t}^{-1}(P(t-1)) P_{d} A_{t}^{-T}\left(P_{d}\right)+P_{d}
\end{aligned}
$$

where $A_{t}(X)=I+r^{-1}(t) X \varphi(t) \varphi^{T}(t), X \in R^{n \times n}$. The equation above is a discrete Sylvester difference equation. Neglecting the dependence of one of the $A_{t}$ operators on $P(t-1)$ makes it possible to apply theory of linear time-varying systems to the problem of analyzing the solutions to (7).

In (Medvedev, 2004) it is proved that in the Riccati equation (3) written as (7), the $P$-matrix does not diverge, in the sense of some norm, when the input signal is not persistently exciting. The matrix $P_{d}$ is a stationary point of (7) and is as well the tuning parameter. For stationary experimental conditions, a good choice of $P_{d}$ is the stationary solution of the 
Riccati equation for the corresponding Kalman filter. In case of non-stationary data with a known time variance, a scheduled sequence of $P_{d}$ approximating the actual non-stationarity can be used.

When the input signal is persistently exciting, the SGalgorithm converges to $P_{d}$. If the excitation is not sufficient in some directions, it can be shown that a manifold of stationary solutions exists, (Evestedt and Medvedev, 2005). Notice here that all the stationary solutions to (7) within the manifold yield the same Kalman gain $K(t)=P_{d} \varphi(t)$ in (3).

If the Sylvester equation (7) converges to one of the stationary solutions and the excitation remains insufficient, then the solution will not depart from the stationary point. Particulary, if there has been sufficient excitation in the input signal for a significant period of time in the past and the solution has converged to $P_{d}$, it will stay there even if the excitation disappears in some directions. This gives the method good windup properties.

On the negative side, the computational complexity of the SG-algorithm is similar to that of the Kalman filter. However, the solution to (7) can be evaluated element-wise provided the direction of excitation is known beforehand, as it is when the data are periodic.

\section{SIMULATIONS}

In this section, simulations are used to study the properties of some recursive parameter estimation algorithms. The first two examples are included to show that with some basic stationary inputs, white noise and a colored signal, the SG algorithm does not perform worse than the others. Then an example is given to highlight the influence of the choice of $P_{d}$ on the estimation performance of the SG algorithm. Next it is shown that in terms of windup properties the $\mathrm{SG}$ algorithm is the best one. Finally simulations are performed to show how the SG algorithm behaves in terms of set point tracking.

\subsection{White noise input}

This example compares the NLMS, AKFA, SG and the Kalman filter in terms of convergence time to $a$ given echo suppression. The input signal $x(t)$ was zero mean white noise, normally distributed with unit variance. The simulated output signal was generated by a real-world time invariant impulse response, and a Gaussian measurement noise $(\mathrm{SNR}=10 \mathrm{~dB})$ was added. The AKFA was initialized with the same parameters as in (Wigren, 1998), Power $=0.25$ $(-6 \mathrm{~dB}), n=512, m=16, \delta=0.001, f_{1}=1$, $f_{2}=0.07815, \epsilon=10^{-6}$, and $N=512$. The numbers reflect a desired echo suppression of $30 \mathrm{~dB}$ and assumptions on the echo power.

The NLMS algorithm used an adaptation gain of $\mu=$ 0.01954 and $n=512$ to get the same echo suppression as the AKFA. The possibility of using a time varying adaptation gain is not utilized in this comparison. The Kalman filter was initialized similarly to the AKFA. The $Q$-matrix was however manipulated to produce an echo suppression of $30 \mathrm{~dB}$

The SG-algorithm was initialized as the Kalman filter. The $P_{d}$-matrix, was however chosen as the stationary point of the Kalman filter, obtained before- hand through simulations. The performance curves are shown in Fig. 2.

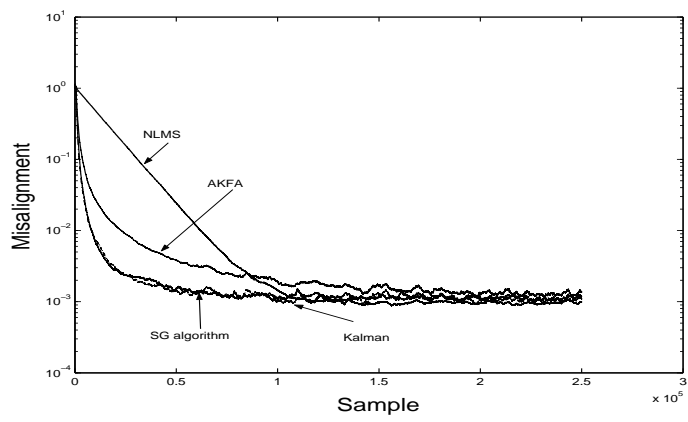

Fig. 2. Convergence time comparison between the NLMS-algorithm, the Kalman filter, AKFA and the SG-algorithm, to a given echo suppression. The measurement of quality is misalignment, defined as $\epsilon=\frac{|h-\hat{h}|}{|h|}$

As can be seen above, the performance of the SGalgorithm, with a proper choice of $P_{d}$, is similar to that of the Kalman filter. The convergence time of the AKFA is, as expected, somewhere between the NLMS and the Kalman filter while its transient response is of the same character as that of the SG-algorithm and the Kalman filter.

\subsection{Colored input}

This example compares the NLMS, AKFA, SG and the Kalman filter when the input signal is colored (11s of music, (Cale, 1976)). Music is a typical signal in this kind of experiments since it usually includes both foreground and background sounds as well as voice. The output signal was generated using a 512tap real world time invariant impulse response and white Gaussian noise was added $(\mathrm{SNR}=10 \mathrm{~dB})$. The AKFA was tuned as in the white noise case. The NLMS-algorithm and the Kalman filter had the same parameter settings as in the previous case. The SGalgorithm used three different constant $P_{d}$-matrices, obtained at three different times from simulations with the Kalman filter. A result of the simulation is given in Fig. 3.

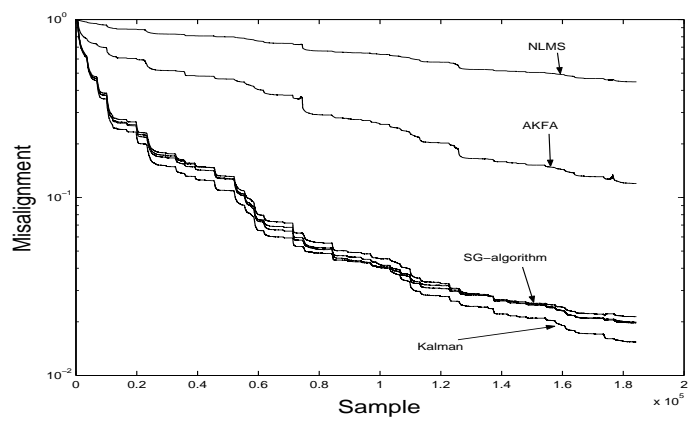

Fig. 3. Estimation accuracy of the NLMS-algorithm, the Kalman filter, AKFA and the SG-algorithm using a non-stationary input (music) and different choices of $P_{d}$.

In this case the Kalman filter performs the best, which should be expected considering the colored input. The AKFA performs significantly better than the NLMS algorithm. The SG algorithm performs similarly to the Kalman filter all three different choices of $P_{d}$. 


\subsection{Piecewise stationary input}

This example provides some insight into the importance of the choice of the $P_{d}$ matrix. The input signal toggled between two zero mean, white noise processes, $e_{1}(t)$ and $e_{2}(t)$ with different variances, $\sigma_{1}$ and $\sigma_{2}$,

$$
x(t)=\left\{\begin{array}{rr}
e_{1}(t) & 1<t \leq 10^{5} \\
e_{2}(t) & 10^{5}<t \leq 1.5 \cdot 10^{5} \\
e_{1}(t) & 1.5 \cdot 10^{5}<t \leq 2 \cdot 10^{5} \\
e_{2}(t) & 2 \cdot 10^{5}<t \leq 2.5 \cdot 10^{5} \\
e_{1}(t) & 2.5 \cdot 10^{5}<t \leq 3 \cdot 10^{5}
\end{array}\right.
$$

The output signal was generated as in the colored input example, with a truncated impulse response of 64 taps. The initial guess of $P$, for the Kalman filter based algorithms, was chosen similarly to the AKFA in the previous examples. The SG algorithm used the stationary solution to the Riccati equation with the different input signals, $e_{1}(t)$ and $e_{2}(t)$, as the choice for $P_{d}$.

Three different simulations were performed. The first one compares the Kalman filter with the SG algorithm, when it is known beforehand when the input signal shifts. At each such shift the $P_{d}$ matrix is also changed accordingly. The results are shown in Fig. 4. As can be

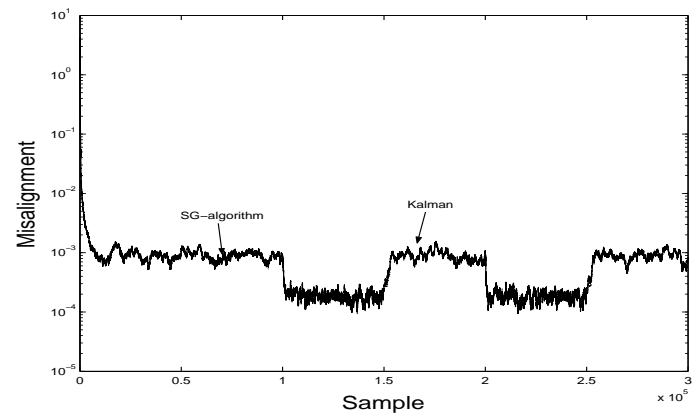

Fig. 4. Estimation accuracy of the SG algorithm and the Kalman filter using a piecewise stationary input with scheduled $P_{d}$. The curves are virtually indistinguishable.

seen the performance of the two algorithms is similar in this case.

If the $P_{d}$ matrix is instead set constant, the results obtained are shown in Fig. 5.

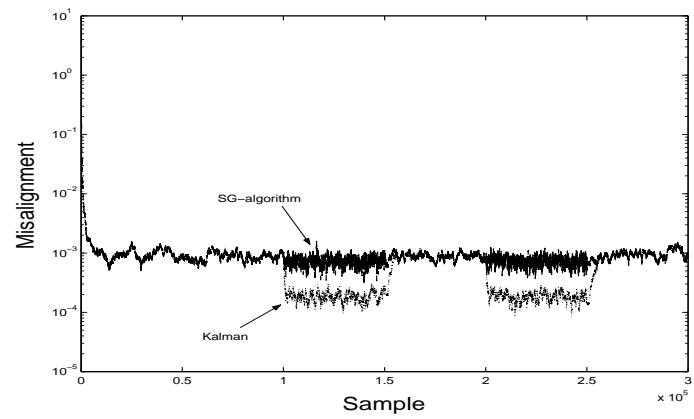

Fig. 5. Estimation accuracy of the SG algorithm and the Kalman filter using a piecewise stationary input with constant $P_{d}$

The figures above indicate that the choice of $P_{d}$ in the SG algorithm is important to the estimation performance. If it is known beforehand that the input signal will be piecewise stationary, solving the algebraic
Riccati equation for each period of stationarity gives the best choice of $P_{d}$ at each stationarity interval.

\subsection{Input signal with decaying energy}

In this example the same $11 \mathrm{~s}$ of music as in the example with colored noise were used as input signal. The energy in the signal was however decreased over time to investigate the algorithms' robustness to windup.

The first seconds were white noise with unit variance to provide the algorithms with initial sufficient excitation. The output was created using the 64 tap impulse response used in the previous example. The initial guess of $P$ was chosen as in the previous examples.

The goal with this setup was to compare how the NLMS, the AKFA, the SG algorithm, the Kalman filter and the method referred to as Algorithm 1 in (Cao and Schwartz, 2004), react to partial loss of excitation. In Fig. 6 the misalignment of the estimated parameters is shown. In Fig. 7 the condition number of the $P$ matrix for the Kalman based algorithms is depicted.

As can be seen, the SG algorithm performs the best and it also has a reasonable constant condition number of the $P$ matrix. The misalignment achieved with the Kalman filter gets worse with time as the signal energy decays. The condition number of the $P$ matrix in the Kalman filter also grows. The NLMS algorithm performs worst when the energy decreases. Algorithm 1 achieves an estimation accuracy in between the NLMS and the Kalman filter.

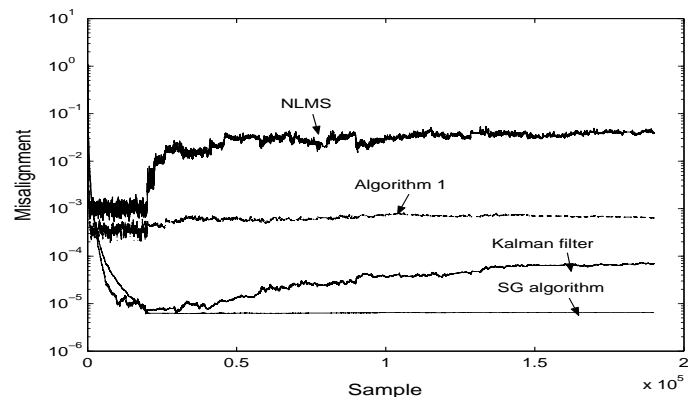

Fig. 6. Misalignment for the recursive parameter estimation algorithms when the input signal energy decreases.

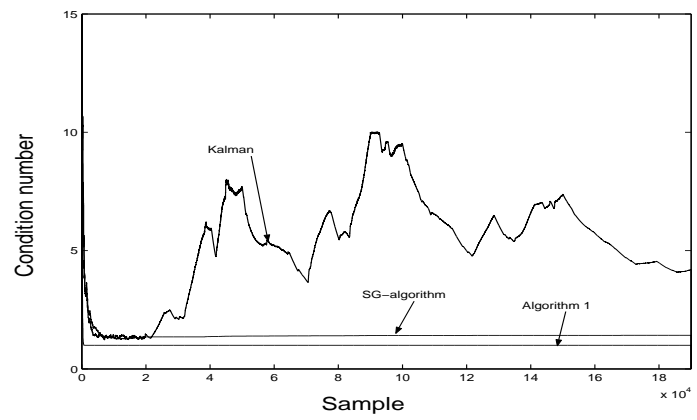

Fig. 7. The condition number of $P$ for the Riccati equation based recursive parameter estimation algorithms when the input signal energy decreases. 


\subsection{Tracking}

In this example the tracking performance of the SG algorithm is studied. The input signal was zero mean white noise with variance $\sigma=5$. The output signal was created using the 64 tap impulse response used in the previous example and the $P$-matrix was initialized as the AKFA. If the $P_{d}$ matrix is considered as a reference signal to the $\mathrm{SG}$ algorithm, the tracking time is defined as the time for the algorithm to converge to a specific $P_{d}$. Here it is illustrated by simulating a change of $P_{d}$ at time $t=50000$. The results are shown in Fig. 8. As can be seen in the figure,

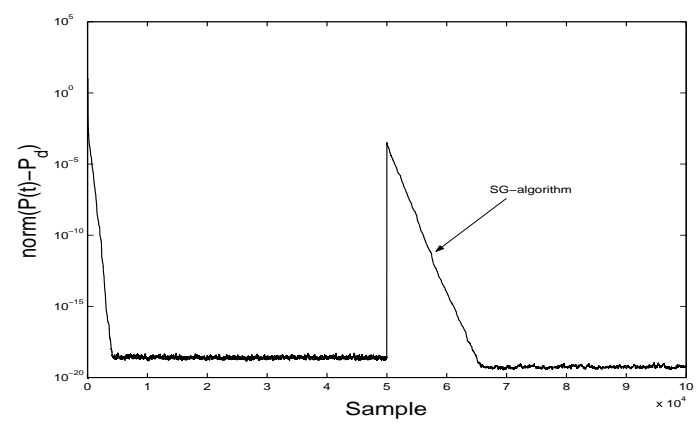

Fig. 8. Tracking performance of the $\mathrm{SG}$ algorithm to a specific $P_{d}$, when changed at $t=50000$. Notice logarithmic scale.

the algorithm converges exponentially, as the Kalman filter (Goodwin and Sin, 1984).

It can be shown that if the excitation is nonpersistent, there is a manifold of stationary solutions. To illustrate this the input signal was chosen as a constant the first 50000 samples. Fig. 9 shows the simulation results. When the input signal is not persistently exciting,

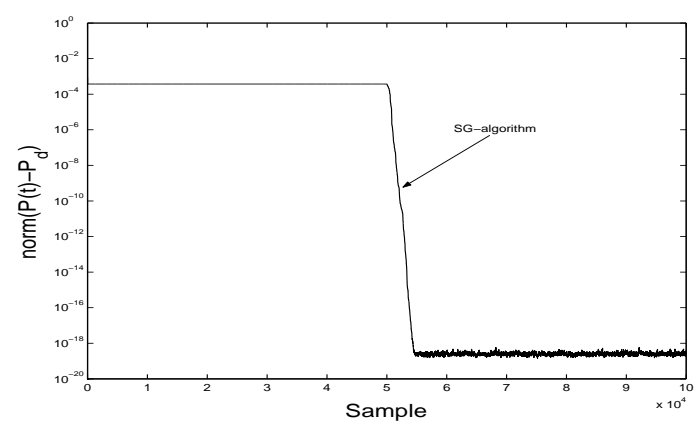

Fig. 9. Tracking performance of the SG algorithm when the input signal is not persistently exciting.

the difference between the stationary solution and $P_{d}$ is constant. When the excitation is recovered, the solution however converges to the matrix $P_{d}$.

\section{CONCLUSIONS}

The windup properties of a recursive parameter estimation algorithm, referred to as the Stenlund Gustafsson (SG) algorithm are studied. As a benchmark for comparing the properties of different recursive parameter estimation algorithms, an acoustic echo cancellation application is used.

The SG algorithm is shown to perform similarly to the Kalman filter in terms of convergence to a given echo suppression with white noise input. With a colored input signal, the accuracy of the estimates achieved by the SG algorithm is close to that of the Kalman filter. The importance of the choice of tuning parameters in the $\mathrm{SG}$ algorithm is highlighted by simulations with a piecewise stationary input signal. The algorithm is shown by simulation to converge exponentially to a pre-defined set point. When the energy in the input signal decreases, the SG estimate has the best accuracy. The windup properties are characterized by the condition number of the solution to the Riccati equation. Once converged to a specific steady-state point, the Riccati equation of the SG-algorithm stays there even when the excitation is insufficient. This gives the method good anti-windup properties.

\section{ACKNOWLEDGEMENTS}

This work has been in part supported by the EC 6th Framework programme as a Specific Targeted Research or Innovation Project (Contract number NMP2CT-2003-505467)

\section{REFERENCES}

Breining, C., P. Dreiseitel, E. Hänsler, A. Mader, B. Nitsch, H. Puder, T. Schertler, G. Schmidt and J. Tilp (1999). Acoustic echo control. IEEE Signal Processing Magazine.

Cale, J.J. (1976). Cocaine. In: Troubadour.

Cao, L. and H.M. Schwartz (2004). Analysis of the Kalman filter based estimation algorithm: an orthogonal decomposition approach. Automatica 40, 5-19.

Evestedt, M. and A. Medvedev (2005). Stationary behavior of an anti-windup scheme for parameter estimation under lack of excitation. In: Proceedings of the 16th IFAC World Congress.

Goodwin, G.C. and K.S. Sin (1984). Adaptive filtering prediction and control. Englewood Cliffs: Prentice-Hall.

Gunnarsson, S. (1996). Combining tracking and regularization in recursive least squares identification. In: Proceedings of the 35th IEEE Conference on Decision and Control. pp. 2551-2552.

Hänsler, E. (1992). The hands-free telephone problem - an annotated bibliography. Signal Processing 27(3), 259-271.

Ljung, L. and S. Gunnarsson (1990). Adaptation and tracking in system identification - a survey. Automatica 26(1), 7-21.

Medvedev, A. (2003). Stability of a windup prevention scheme in recursive parameter estimation. In: Proceedings of the 42nd IEEE Conference on Decision and Control.

Medvedev, A. (2004). Stability of a Riccati equation arising in recursive parameter estimation under lack of excitation. IEEE Transactions on Automatic Control 49(12), 2275-2280.

Stenlund, B. and F. Gustafsson (2002). Avoiding windup in recursive parameter estimation. In: Preprints of reglermöte 2002.

Wigren, T. (1998). Fast converging and low complexity adaptive filtering using an averaged Kalman filter. IEEE Transactions on Signal Processing 46(2), 515-518. 